\title{
Ambient networks and services
}

\author{
Elhadi Shakshuki • Muhammad Younas • \\ Tarek Sheltami
}

Published online: 21 June 2011

(c) Springer-Verlag 2011

Ambient networks are considered as modern mobile networks that involve heterogeneous mobile devices, networks and users. The distinguishing feature of ambient networks is that they allow for dynamic composition of different networks, systems and services. Such composition enhances the capability of networks and also provides services that suit the users and the network environments. The research community has actively investigated the underlying challenges pertaining to these networks, as they fundamentally differ from the classical problems due to their inherent constraints.

The theme of this special issue is to present latest research on ambient networks, systems and services. This special issue includes a selection of papers from an open call as well as selected papers from the International Conference on Ambient Systems, Networks and Technologies (ANT 2010) which was held in November 2010 in Paris, France and has attracted excellent scientific papers contributing to the state of the art in this research field. All the papers were subjected to a two rounds of rigorous review process. Based on the reviews, a total of seven papers were selected for publication. These papers present

\footnotetext{
E. Shakshuki $(\bowtie)$

Jodrey School of Computer Science, Acadia University,

Wolfville, Canada

e-mail: elhadi.shakshuki@acadiau.ca

M. Younas

Department of Computing and Electronics,

Oxford Brookes University, Oxford, UK

e-mail: m.younas@brookes.ac.uk

T. Sheltami

Computer Engineering Department, King Fahad University,

Dhahran, Saudi Arabia

e-mail: tarek@kfupm.edu.sa
}

interesting research work on ambient networks and intelligence, wireless and sensor networks, and mobile services and applications.

Self-organisation is an important research issue in ambient networks. Habib and Marimuthu exploit molecular biology assembly techniques in the self-organisation of ambient networks. This approach analogizes the ambient network nodes as molecules in order to form clusters of such nodes in a self-organised manner. Clusters are formed by defining pair-wise relations between nodes such as the distance, incoming traffic and outgoing traffic. Depending on the type of relationship, nodes attract (or repulse) each other to (from) the cluster. The experimental results show that the proposed approach significantly optimises backbone traffic.

The paper by Buchmayr and Kurschl surveys the existing projects and developments in the situation- aware ambient intelligence. The aim of the survey is to establish the knowledge and understanding of the large number of prototypes and working systems that have been developed for situation-aware ambient intelligence. Based on the survey, this paper designs a reference architecture and also develops an evaluation criteria for situation awareness in ambient systems. The survey also identifies open research issues for new research projects in the area of ambient intelligence.

Becerra and Kremer propose a multi-agent approach in order to design an ambient intelligent environment. This work considers an interesting application area wherein agents are used to represent and control various inhabitants such as humans, animals, plants and objects such that they can sacrifice their own needs for the benefit of the whole environment. For instance "a dark room could prevent the plants from growing; a cold room would make the leather sofa uncomfortable to sit on when the man returns; 
a warm, bright room could cause algae in the aquarium or damage the paintings". The proposed approach aims to provide a balanced environment by taking into account the individual inhabitant needs as well as the environment needs as a whole. The experimental indicates that it is possible to achieve a balance by having each inhabitant receive sub-optimal environmental conditions, but the environment as a whole achieved near-optimal level.

Ambient intelligence is also crucial to the effective management of information in real life application areas such as healthcare, traffic monitoring, weather information. De Amicis et al. exploit ambient intelligence and serviceoriented computing in the management of transport infrastructure. This work has practical applications and has been developed for a major Italian motorway operator. In it, the authors develop a system that performs complex activities involved in the transport infrastructure of motorways. The system can be used to access and manage useful information on geographical data, traffic routes, tollbooths, traffic data and weather information among others. Such information is also used by general public in order to know about traffic congestion, weather condition and road side emergencies.

In relation to the transport infrastructure, Delot et al. investigate into the multi-scale query processing of vehicular networks. Such networks are highly mobile with vehicles as nodes that travel along a road and communicate using short range wireless communication devices. The underlying approach is to combine different access modes in order to retrieve data from different data sources such as local data or data from other vehicles. The objective is enable users to execute queries such as "Retrieve the list of petrol stations located in a radius of $10 \mathrm{~km}$ around me where fuel prices are less than $\$ 1$ (updating the result every $5 \mathrm{~min}$ )". This work is validated through a prototype using the Microsoft LINQ API.

Malik et al. propose a methodology for optimising queries in wireless sensor networks. The proposed methodology, based on the historical data, can be used to construct optimal queries. Such optimization is achieved through reducing the number of sensory attributes involved in a query. The proposed methodology is validated through real world sensor data and the results obtained show greater accuracy in the processing of queries.

Collaborative activities have been widely researched in classical/stationary systems. However, little attention has been given to such area in the domain of mobile systems. Komaki et al. propose an interesting approach for collaborative web search using mobile devices. The proposed approach provides a group of mobile device users with facilities of searching the web collaboratively. It develops an interface system that enables users to collaboratively share and compare the search results while using multiple mobile devices. Potential benefits of this work are to simplify mobile web searching, reduce participants' subjective operations and to enable group discussion and communication.

The guest co-editors would like to take this opportunity to thank the authors for the efforts they put in the preparation of their manuscripts and for their valuable contributions. We wish to express our deepest gratitude to the referees for their thorough detailed reviews. Last, but not least, our sincere thanks go to the Editor-in-Chief Professor Vincenzo Loia of the Journal of Ambient Intelligence and Humanized Computing for the exceptional support and assistance he provided throughout this process. 